\title{
Ovarian thecoma with massive pleural effusion in postmenopausal women: A case report
}

\author{
YANG TING, LI YANG, ZHAO JUAN, WEI XING and YANG XIAO FENG \\ Department of Obstetrics and Gynecology, The First Affiliated Hospital of Xi'an Jiaotong University, \\ Xi'an, Shaanxi 710061, P.R. China
}

Received December 15, 2015; Accepted March 18, 2016

DOI: $10.3892 / \mathrm{mco} .2016 .853$

\begin{abstract}
Previous clinical reports of benign ovarian thecoma, associated with hydrothorax, are rare. The present study presents the case of a 58-year-old women exhibiting right massive pleural effusions and elevated cancer antigen (CA)125, and a pelvic ultrasound revealed a hypoechoic mass. An exploratory laparotomy was subsequently performed. During the surgery, the left ovary measured $20 \times 15 \times 15 \mathrm{~cm}$ and had a smooth surface with no excrescences or papillary projections. The patient underwent right salpingo-oophorectomy. Pathological diagnosis was determined as benign ovarian thecoma. The chest radiograph revealed no pleural effusion 6 days following the surgery. The present case demonstrated a benign ovarian thecoma, associated with massive hydrothorax and elevated CA125, which mimicked an ovarian malignancy.
\end{abstract}

\section{Introduction}

Ovarian thecoma is a rare benign tumor of stromal cell origin, and represents $<1 \%$ of all ovarian tumors (1). It occurs most often in perimenopausal and postmenopausal women. Elevated serum cancer antigen (CA)125 levels in postmenopausal women with solid adnexal masses, ascites and pleural effusion are highly suggestive for malignant ovarian tumors. However, surgery and histological confirmation of the preoperative diagnosis are mandatory, since a minority of patients with these findings have a benign condition, commonly known as Meigs' syndrome. This condition disappears following the removal of the pelvic tumor. The present study reported a case of massive pleural effusions caused by right ovarian thecoma with elevated serum CA125 levels in a postmenopausal woman. This patient was clinically interpreted to harbor a malignant ovarian tumor, which was eventually revealed to be benign ovarian thecoma.

Correspondence to: Dr Yang Xiao Feng, Department of Obstetrics and Gynecology, The First Affiliated Hospital of Xi'an Jiaotong University, 277 West Yanta Road, Xi'an, Shaanxi 710061, P.R. China E-mail: yxf73@163.com

Key words: ovarian thecoma, pleural effusion, CA125

\section{Case report}

A 58-year-old, para 2 women visited the Respiratory Medical Clinic at The First Affiliated Hospital of Xi'an Jiaotong University (Shaanxi, China) in December 2014 due to a 1-week-long cough and shortness of breath for 4 days. The patient went through the menopause at the age of 50. Chest examination revealed dullness to the percussion and diminished breath sounds on the right lung. Chest radiography revealed right pleural effusions (Fig. 1A). The patient underwent thoracentesis, followed by draining of yellowish pleural effusion $(5,000 \mathrm{ml})$ by closed thoracic drainage. Pleural fluid cytology revealed no malignant cells. The CA125 tumor marker level was reported as $577 \mathrm{IU} / \mathrm{ml}$ (normal, <35 U/ml), and other studies, including respiratory virus antibody, Mycobacterium tuberculosis antibody immunoglobulin G, tuberculosis-DNA, tuberculosis spot test, endotoxin and fungal glucan, revealed negative findings. A pelvic ultrasound revealed a hypoechoic mass measuring $16.5 \times 13.8 \times 13 \mathrm{~cm}$ and the uterus endometrium thickness of $0.4 \mathrm{~cm}$, with some pelvic ascites $(6.2 \times 2.7 \mathrm{~cm})$. The patient was finally referred to the Department of Obstetrics and Gynecology, The First Affiliated Hospital of Xi'an Jiaotong University, (Shaanxi, China) as a result of this mass. Following the initial hypothesized diagnosis of an ovarian tumor with ascites and hydrothorax, an exploratory laparotomy was performed. During the operation, the left ovary measured 20x15x15 cm and had a smooth surface with no excrescences or papillary projections. The left tube was elongated along the course of the distended ovary, The right ovary was small with no evidence of cysts. The right fallopian tube was normal and the uterus was normal. No evidence of ascites or peritoneal deposits were observed, and the surfaces of the bowel, liver and omentum were normal. No pelvic or para-aortic nodal enlargement was observed. The patient underwent a right salpingo-oophorectomy and the frozen section appeared to be benign.

Upon gross examination, the ovary measured $17 \times 14 \times 6 \mathrm{~cm}$ and no surface excrescences or nodules were observed. The cyst itself was multiloculated and contained clear, watery serous-type fluid, with a smooth inner wall of gray. The length of fallopian tube was $2.5 \mathrm{~cm}$, the diameter was $0.5 \mathrm{~cm}$ and the oviduct fimbria was opening. Microscopic images of the tumor revealed uniform and cytologically bland-looking spindle 

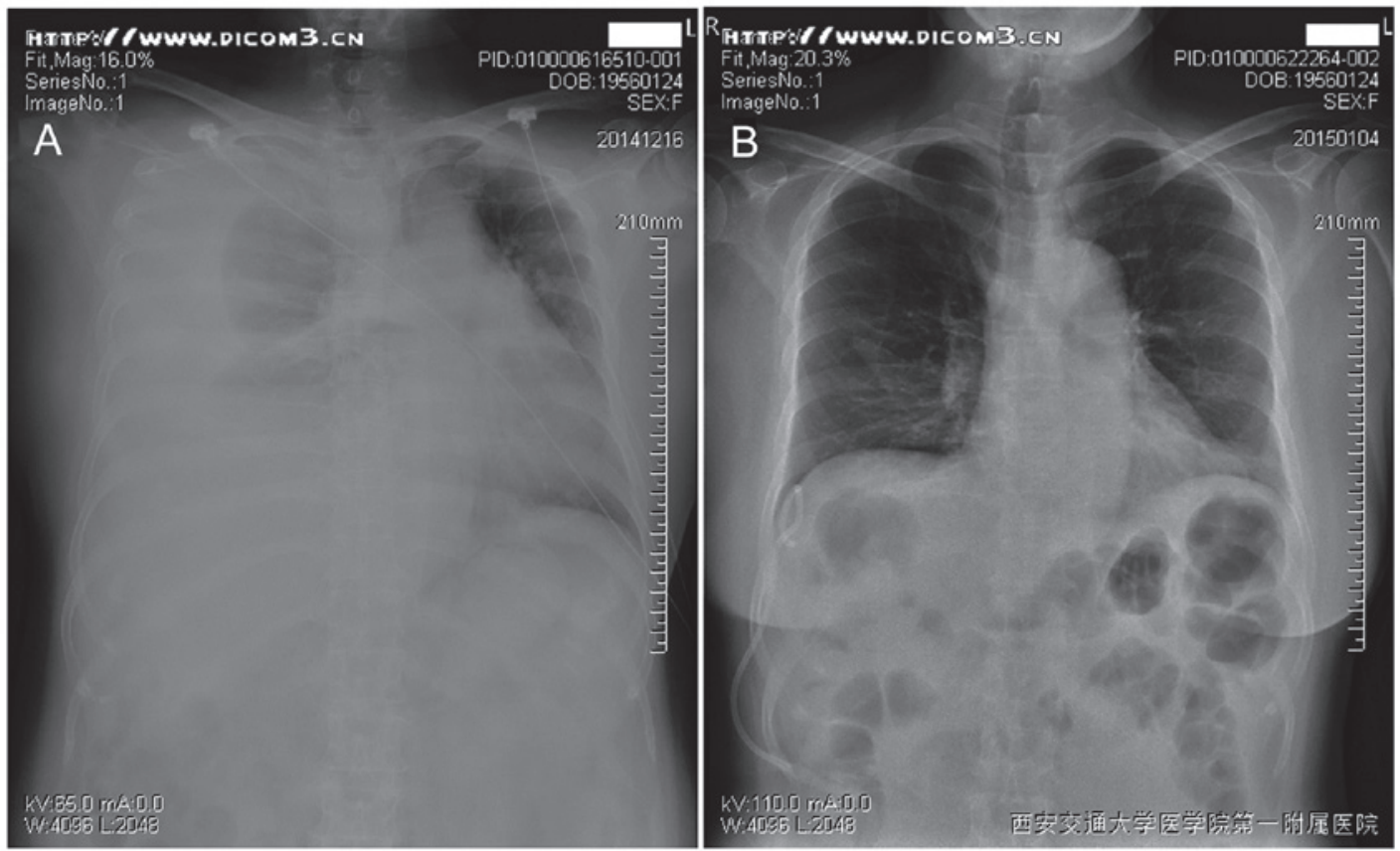

Figure 1. (A) A chest radiograph revealed right mass pleural effusions. (B) The chest radiograph revealed no pleural effusion six days following surgery.
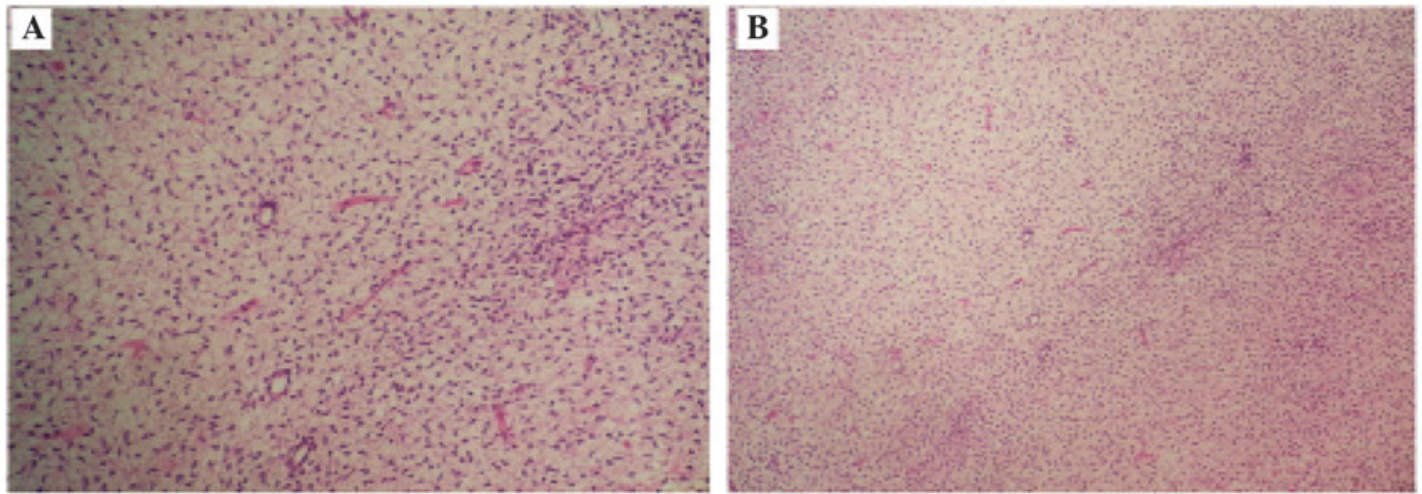

Figure 2. Histopathological examination of the tumor by hematoxylin and eosin staining. (A) Thecoma was represented by long fascicles in a storiform and whorl arrangement (magnification, x40). (B) Staining also revealed uniform and cytologically bland-looking spindle cells arranged in fascicles (magnification, x10).

cells arranged in fascicles. Pathological diagnosis was benign ovarian thecoma (Fig. 2). The chest radiograph revealed no pleural effusion even 6 days after the surgery (Fig. 1B).

\section{Discussion}

Thecomas make up only $0.5-1 \%$ of ovarian tumor types. They occur mostly in postmenopausal women, with a mean age of 59 years, and only $10 \%$ of the patients are normally $<30$-years-old. Thecomas range from small tumors to large solid or solid-cystic masses of up to $15 \mathrm{~cm}$. They are unilateral in $>90 \%$ of cases and are rarely malignant $(2,3)$. Thecomas are stromal tumors made up of cells that resemble theca cells, lutein cells and fibroblasts (4). They are traditionally classified within the sex cord-stromal tumor category of ovarian tumor types (5).

In the present case, the patient was admitted to the Respiratory Department at The First Affiliated Hospital of Xi'an
Jiantong University due to breathlessness with no abdominal pain, bloating and other symptoms. It was revealed that the expression of CA-125 was increased, and then a reproductive system tumor was considered. CA125 has been demonstrated to be elevated in benign conditions, including pelvic inflammatory disease, endometriosis, uterine leiomyoma and early pregnancy. The serum CA125 levels are also increased with peritoneal pleural and pericardial inflammation or irritation. A certain misdiagnosis rate occurs with Hydroperitonia theca cell tumors.

In 1954 Meigs reviewed 122 cases of abdominal or pelvic lesions with ascites and hydrothorax, and found 84 typical cases of Meigs' syndrome (6). The importance of Meigs' syndrome is that the presence of ascites and pleural effusion does not necessarily indicate that a pelvic mass is malignant. The benign tumors in Meigs syndrome are usually fibromas or fibrothecomas, and constitute $4 \%$ of all ovarian neoplasms (7). Others include mucinous cystadenomas and Brenner tumors of the ovary (8). 
Ascites probably occurs by means of a transudative mechanism through the tumor surface, which exceeds the peritoneum's resorptive capacity (9). Other possible mechanisms include obstruction of peritoneal lymphatics by the tumor or increased permeability of the neovasculature with protein leakage. From the basic biological point of view, inflammatory cytokines are known to induce capillary leakage and third-space fluid accumulation in numerous gynecologic and non-gynecologic disorders. The cytokines, including interleukin (IL)-1 $\beta$, IL-6, IL-8 and tumor necrosis factor (TNF)- $\alpha$ in the interstitial fluid of Meigs' syndrome with ascites and hydrothorax were investigated, and they all decreased following tumor removal, with the exception of TNF- $\alpha$ (10).

The present study reported an uncommon case of a benign ovarian thecoma, associated with hydrothorax, which mimicked an ovarian malignancy. Surgery is the preferred treatment for thecoma, and the present study suggested that the patient accept the hysterectomy and bilateral salpingo-oophorectomy in order to avoid endometrial cancer in postmenopausal women. However, salpingo-oophorectomy may be used for older or weaker patients. Increased attention must be paid to determine the surgical procedures used, due to the common ovarian thecoma combined with endometrial cancer.

\section{References}

1. Takemori M, Nishimura R and Hasegawa K: Ovarian thecoma with ascites and high serum levels of CA125. Arch Gynecol Obstet 264: 42-44, 2000.

2. Chen VW, Ruiz B, Killeen JL, Coté TR, Wu XC and Correa CN: Pathology and classification of ovarian tumors. Cancer 97 (Suppl 10): S2631-S2642, 2003.

3. Nocito AL, Sarancone S, Bacchi C and Tellez T: Ovarian thecoma: Clinicopathological analysis of 50 cases. Ann Diagn Pathol 12: 12-16, 2008.

4. Tavassoli FA, Mooney E and Gersell DJ: Sex cord-stromal tumors. In: Tumors of the breast and female genital organs. Tavassoli FA and Deville P (eds). IARC Press, Lyon, pp149-151, 2003.

5. Scully RE, Young RH and Clement PB: Stromal tumors. In: Tumors of the ovary, maldevelopment gonads, fallopian tube and broad ligament. Atlas of tumor pathology. Rosai J (ed). Armed Forces Institute of Pathology, Washington, pp189-197, 1998.

6. Meigs JV: Fibroma of the ovary with ascites and hydrothorax; Meigs' syndrome. Am J Obstet Gynecol 67: 962-985, 1954.

7. Nemeth AJ and Patel SK: Meigs syndrome revisited. J Thorac Imaging 18: 100-103, 2003.

8. Buttin BM, Cohn DE and Herzog TJ: Meigs' syndrome with an elevated CA 125 from benign Brenner tumors. Obstet Gynecol 98: 980-982, 2001.

9. Samanth KK and Black WC III: Benign ovarian stromal tumors associated with free peritoneal fluid. Am J Obstet Gynecol 107: $538-545,1970$.

10. Abramov Y, Anteby SO, Fasouliotis SJ and Barak V: The role of inflammatory cytokines in Meigs' syndrome. Obstet Gynecol 99: 917-919, 2002. 